\title{
Partial duplications of the ATRX gene cause the ATR-X syndrome
}

\author{
Bernard Thienpont ${ }^{1}$, Thomy de Ravel ${ }^{1}$, Hilde Van Esch ${ }^{1}$, Dominique Van Schoubroeck ${ }^{2}$, \\ Philippe Moerman ${ }^{3}$, Joris Robert Vermeesch ${ }^{1}$, Jean-Pierre Fryns ${ }^{1}$, Guy Froyen ${ }^{4}$, \\ Caroline Lacoste ${ }^{5}$, Catherine Badens ${ }^{5}$ and Koen Devriendt*,1
}

${ }^{1}$ Center for Human Genetics, Catholic University of Leuven, Leuven, Belgium; ${ }^{2}$ Department of Obstetrics, University Hospital Leuven, Leuven, Belgium; ${ }^{3}$ Department of Pathology, University Hospital Leuven, Leuven, Belgium; ${ }^{4}$ Human Genome Laboratory, Centre for Human Genetics, Flanders Interuniversity Institute for Biotechnology, Leuven, Belgium; ${ }^{5}$ Laboratoire de Génétique Moléculaire, Département de Génétique Médicale, Hôpital d'enfant de la Timone, Marseille, France

ATR-X syndrome is a rare syndromic X-linked mental retardation disorder. We report that some of the patients suspected of ATR-X carry large intragenic duplications in the ATRX gene, leading to an absence of $A T R X$ mRNA and of the protein. These findings underscore the need for including quantitative analyses to mutation analysis of the ATRX gene.

European Journal of Human Genetics (2007) 15, 1094-1097; doi:10.1038/sj.ejhg.5201878; published online 20 June 2007

Keywords: ATRX; intragenic duplication; mutation; array-CGH

\section{Introduction}

ATR-X syndrome is a rare $\mathrm{X}$-linked syndrome characterized by severe to profound mental retardation, characteristic facial appearance, genital anomalies and alpha thalassaemia. ${ }^{1}$ A mutation is identified only in a subset of patients with clinical suspicion of ATR-X syndrome. In this report, we show that some of the patients suspected of having ATR$\mathrm{X}$ syndrome but negative for mutation analysis, carry a large duplication inside $A T R X$, thereby extending the spectrum of mutation associated with this disorder. In one patient, we show that this duplication causes a severe reduction of ATRX mRNA and absence of the ATRX protein.

\section{Case reports}

Family 1

Patient 1a was born at term after a normal pregnancy. There was peripartal asphyxia. Biometry was at the 50th centile. The neonatal period was complicated by convulsions and

*Correspondence: Professor K Devriendt, Center for Human Genetics, Catholic University of Leuven, Herestraat 49, B-3000 Leuven, Belgium. Tel: + 3216 345903; Fax: + 3216 346060;

E-mail: koenraad.devriendt@uzleuven.be

Received 11 January 2007; revised 10 May 2007; accepted 22 May 2007; published online 20 June 2007 severe hypotonia. There was hypertelorism, low set ears, inverted nipples and female external genitalia with small labia minores. Brain MRI was normal and he had an atrial septum defect type II. Karyotype was normal male, 46,XY in white blood cells and skin fibroblasts. The child died at the age of 4.5 months from hypoventilation. Autopsy confirmed true male hermaphroditism with absent uterus and two dysgenetic testes. The gallbladder was absent.

During the second pregnancy, ultrasound examination at 15 weeks gestation indicated a female appearance of the external genitalia. Amniocentesis showed a normal male, 46,XY karyotype (patient 1b). At 19 weeks of gestation, ultrasound confirmed the female appearance of the external genitalia and detected an atrial septal defect. The pregnancy was interrupted. Ambiguous genitalia, with a severe hypospadias, and a micropenis were present. Necropsy confirmed the presence of a large ASDII. There was no uterus and the gonads were intra-abdominal. Also, the gallbladder was absent and bilateral bilobar lungs were noted. The normal male karyotype was confirmed on cultured fetal skin fibroblasts.

\section{Family 2}

Patient 2 was born at term after caesarean section for prolonged labour. APGAR score was 6 after $10 \mathrm{~min}$. Birth 
weight was $3.2 \mathrm{~kg}$ (25-50th centile), length $53.5 \mathrm{~cm}$ (5075 th centile) and head circumference $35 \mathrm{~cm}$ (50th centile). He needed nasogastric tube feeding and there was failure to thrive. At age 6 months, weight was $4.53 \mathrm{~kg}$ (3rd centile $=5.3 \mathrm{~kg}$ ), length $61 \mathrm{~cm}$ (3rd centile) and head circumference $39.2 \mathrm{~cm}$ (3rd centile $=40 \mathrm{~cm}$ ). There was cryptorchidism, a small penis and bilateral clinodactyly of the fifth fingers. He had anteversion of the nostrils, a broad columella and hypertelorism with epicanthic folds. Karyotype was normal male 46,XY. Development was profoundly delayed with severe axial hypotonia and peripheral hypertonia. MRI scan of the brain revealed agenesis of the corpus callosum. He had a sensorineural hearing loss of $50 \mathrm{~dB}$ and suffered from chronic unexplained anaemia. Cardiac ultrasound was normal. He died unexpectedly at age 20 years.

\section{Materials and methods}

Array-CGH was performed as described using a microarray containing BAC/PAC clones with a genome-wide $1 \mathrm{Mb}$ resolution (donated by the Sanger Institute, Hinxton, UK), or with a $80 \mathrm{~kb}$ resolution for the $\mathrm{X}$ chromosome. ${ }^{2,3}$ Realtime quantitative PCR (QPCR) was performed as described ${ }^{2}$ using primers designed inside, upstream and downstream of ATRX (Figure 1b, Supplementary Table 1). The X inactivation pattern was determined as described. ${ }^{4}$

Total RNA was extracted using the RNeasy Mini Kit (Qiagen) and after DNaseI (Roche) treatment, it was reverse transcribed with Superscript II (Invitrogen) using a mixture of random and poly-A oligonucleotide primers. QPCR on cDNA (RT-PCR) was performed using specific primers designed in exons 1 and 2 of ATRX (Supplementary Table 1). ATRX mRNA level was quantified by comparison to mRNA levels of genes showing stable expression (GAPD, $\beta 2 M$ and $\beta$-act $).^{5}$

Nuclear protein fractions were extracted as described. ${ }^{6}$ Twenty micrograms per lane was submitted to Western blotting. The ATRX protein was detected using the monoclonal antibody 23C, ${ }^{6}$ a gift from Dr Garrick (WIMM, John Radcliffe Hospital, Oxford, UK). Detection of SP1 (PEP2, sc-59, Santa Cruz) using a polyclonal antibody served as a positive control for nuclear protein extraction.

\section{Results}

In patient $1 \mathrm{a}$, array-CGH at $1 \mathrm{Mb}$ resolution revealed the duplication of two neighbouring clones located on the $\mathrm{X}$ chromosome (RP5-875J14 and RP3-465G10). In his sibling (patient 1b), array-CGH using the X-chromosome array revealed two non-contiguous duplications, one inside the ATRX gene, and one upstream (Figure 1a, Supplementary Table 2).

QPCR using primer pairs i1-2, i8-9 and i28-29 on a cohort of 50 patients with suspected ATR-X syndrome but without a detected sequence alteration in this gene revealed the presence of a duplication in one additional patient (patient 2). Array-CGH using the X-chromosome microarray confirmed the presence of the duplication in patient 2 (Figure 1a, Supplementary Table 2). Consecutive rounds of QPCR using primer pairs e1, i1 -2, e2, i2-3, i8-9, i28-29, e29, e30, e35 and e36 (Figure 1b) demonstrated that both duplications have different breakpoints. They span exons $2-35$ in family $1(222-281 \mathrm{~kb})$ and exons $2-29$ in family 2 (143-184 kb) (Figure 1c).

Further analysis by QPCR in the families indicated that both mothers carry the ATRX duplications. In family 1, the maternal grandparents did not carry the duplication. Likewise, in family 2 , the healthy brother and sister of the index patient had no duplication.

Both carrier mothers had completely skewed X-inactivation (family 1: 100\%, family 2: 99.35\%). Analysis of the polymorphic $A R$ repeat $^{7}$ (located approximately $11 \mathrm{Mb}$ from $A T R X)$ revealed that both patients $1 \mathrm{a}$ and $1 \mathrm{~b}$ carried the same allele, located on the inactivated $X$ chromosome of their mother. She inherited this chromosome from her father, indicating the duplication occurred in the grandfather. Also patient 2 and his mother's inactivated $\mathrm{X}$ chromosome carry the same allele.

To assess the effect of this duplication on the functioning of ATRX, ATRX mRNA levels were quantified in a cell line from patient $1 \mathrm{~b}$, showing a reduction to $3 \%$ of the normal level $(P<0.01$; Figure 1d). RT-PCR on mRNA from his mother showed normal ATRX mRNA levels (data not shown). In addition, Western blot analysis revealed a total absence of the ATRX protein in the fibroblast cell line from patient 1b (Figure 1e).

\section{Discussion}

In a family with two siblings presenting an unexplained disorder with cardiac and genital malformations, array$\mathrm{CGH}$ led to the identification of an intragenic duplication of the ATRX gene. In retrospect, the clinical features are fully compatible with the ATR-X syndrome. Less classical features, including true male pseudohermaphroditism and congenital heart defects, have been described before. ${ }^{1}$ However, we are unaware of previous reports of absent gallbladder in this syndrome.

Extending this study in a cohort of 50 additional patients suspected for ATR-X syndrome led to the identification of one additional patient carrying an intragenic ATRX duplication. Given the position of the duplications, they are expected to result in a loss of function. This was confirmed in skin fibroblasts from one of the patients, patient 1b. RT-PCR showed a drastic reduction of the level of ATRX mRNA resulting in a reduction in the level of ATRX protein below the detection limit of Western blot, demonstrating the detrimental nature of the intragenic 



Figure 1 (a) Array-CGH. Array-CGH analysis on patients 1a $(\triangle)$ and $2(\bigcirc)$ showing an amplification of RP13-728A10 (1.), RP5-875J14 (2.), RP11$42 \mathrm{M} 11$ (3.) and RP3-346O6 (4.) in both patients and an additional duplication from RP3-465G10 to RP13-213F13 in patient 1a. The position of ATRX is indicated below. Patient DNA labelled with Cy5. Array-CGH values are in Supplementary Table 2. (b) The ATRX region. The positions of BAC clones fully or partially duplicated on array-CGH and all QPCR primers used in this study, relative to the ATRX gene (36 exons are indicated, with exon 7 spliced out. accession no. U72937). Primer sequences are listed in Supplementary Table 1. Black and grey bars below the region indicate the certainly and potentially duplicated region, respectively. (c) QPCR. Results of QPCR analysis of three normal males $(N)$, patient 1a and patient 2. (d) mRNA expression. The expression of $A T R X$ mRNA in cultured skin fibroblasts of patient $1 \mathrm{~b}(P)$ is compared to normal $46, X Y$ fetal skin fibroblasts $(N)$ cultured in parallel. Values are normalized to $N$. RC = relative concentration. OPCR on CDNA was performed using primers expr forw and expr rev designed in exons 1 and 2. (e) Western blot. The expression of the ATRX protein in cultured skin fibroblasts of patient $1 \mathrm{~b}(\mathrm{P})$ is compared to that in normal $46, \mathrm{XY}$ fetal skin fibroblasts $(N)$ cultured in parallel. SP1 = a nuclear protein serving as control for nuclear protein extraction. 
duplication. Previous studies similarly showed a drastic reduction or even an apparent loss of the ATRX protein in some mutation carriers. ${ }^{8}$

This observation of an intragenic ATRX duplication leading to gene disruption extends the spectrum of mutations causing the ATR-X syndrome. This has important practical consequences: traditional mutation analysis strategies relying on non-quantitative techniques for sequence analysis will need to be complemented by additional techniques allowing the detection of copy number changes. In other genes checked for gross genomic rearrangements, deletions and duplications are detected but their frequency varies dramatically: deletions are detected in DMD, TSC1, TSC2, CFTR and NF1 with reported frequencies of $65,0.45,6,1.5$ and $2 \%$, while intragenic duplications account for $7,0,0.24,0$ and $0.3 \%$ of mutations. ${ }^{9-13}$ The reason why duplications are in general less frequent is not known. One reason could be that the mechanisms generating duplications are more complex than those generating deletions. Alternatively, certain whole gene duplications lead to a different phenotype than the loss-of-function phenotype associated with whole gene deletions, and will thus not be ascertained, as was seen for example in MECP2. ${ }^{14}$ For the gene in which most duplications were hitherto characterized, $D M D$, no bias towards larger duplications was detected. ${ }^{9}$ Because in the present study patients were not checked for duplications in each of the 36 exons of the ATRX gene, we expect the frequency of duplication mutations may be higher than reported. Also in this perspective, it is somewhat surprising that gross ATRX deletions are not yet detected in ATR-X patients, while we report on two different duplication events, one of which apparently causes loss-of-protein.

Array-CGH detected two flanking non-contiguous duplications in family 1: one inside $A T R X$ and one spanning ATP7A, PGAM4, PGK1 and TAF9B. We cannot exclude that this second duplication contributed to the phenotype of the siblings in family 1 . Although uncommon, recent higher resolution analyses show that this type of complex intrachromosomal rearrangements occur more often than hitherto appreciated. ${ }^{9,15}$ This implies caution for the extrapolation of copy-number measurements in discrete genomic regions to the regions in between. In this study, array-CGH at $1 \mathrm{Mb}$ resolution showed two duplicated clones in family 1. Extrapolation would have implied the duplication of $A T R X$ is not intragenic. Only higher resolution analysis revealed that this were two noncontiguous duplications, with one disrupting ATRX.

In conclusion, the present observation adds a novel type of mutations detectable in the ATRX gene, underscoring that quantitative analyses should be an integral part of mutation analysis in this and other disease genes.

\section{Acknowledgements}

We thank the families for their kind cooperation, Dr D Garrick (Weatherall Institute of Molecular Medicine, Oxford, UK) and I Cleynen (Department for Human Genetics, Leuven, Belgium) for the generous donation of the antibodies, and Dr F Foulquier for sharing his expertise in protein studies.

\section{References}

1 Gibbons R: Alpha thalassaemia-mental retardation X linked. Orphanet J Rare Dis 2006; 1: 15.

2 Menten B, Maas N, Thienpont B et al: Emerging patterns of cryptic chromosomal imbalances in patients with idiopathic mental retardation and multiple congenital anomalies: a new series of 140 patients and review of the literature. J Med Genet 2006; 43: 625-633.

3 Bauters M, Van Esch H, Marynen P, Froyen G: X chromosome array-CGH for the identification of novel X-linked mental retardation genes. Eur J Med Genet 2005; 48: 263-275.

4 Devriendt K, Matthijs G, Legius E et al: Skewed X-chromosome inactivation in female carriers of dyskeratosis congenita. Am J Hum Genet 1997; 60: 581-587.

5 Vandesompele J, De Preter K, Pattyn F et al: Accurate normalization of real-time quantitative RT-PCR data by geometric averaging of multiple internal control genes. Genome Biol 2002; 3: RESEARCH0034.

6 McDowell TL, Gibbons RJ, Sutherland $\mathrm{H}$ et al: Localization of a putative transcriptional regulator (ATRX) at pericentromeric heterochromatin and the short arms of acrocentric chromosomes. Proc Natl Acad Sci USA 1999; 96: 13983-13988.

7 Allen RC, Zoghbi HY, Moseley AB, Rosenblatt HM, Belmont JW: Methylation of HpaII and Hha sites near the polymorphic CAG repeat in the human androgen-receptor gene correlates with $\mathrm{X}$ chromosome inactivation. Am J Hum Genet 1992; 51: 1229-1239.

8 Abidi FE, Cardoso C, Lossi AM et al: Mutation in the $5^{\prime}$ alternatively spliced region of the XNP/ATR-X gene causes Chudley-Lowry syndrome. Eur J Hum Genet 2005; 13: 176-183.

9 White SJ, Aartsma-Rus A, Flanigan KM et al: Duplications in the DMD gene. Hum Mutat 2006; 27: 938-945.

10 White S, Kalf M, Liu Q et al: Comprehensive detection of genomic duplications and deletions in the DMD gene, by use of multiplex amplifiable probe hybridization. Am J Hum Genet 2002; 71 : $365-374$.

11 Kozlowski P, Roberts P, Dabora S et al: Identification of 54 large deletions/duplications in TSC1 and TSC2 using MLPA, and genotype-phenotype correlations. Hum Genet 2007; 121: 389-400.

12 Wimmer K, Yao S, Claes $\mathrm{K}$ et al: Spectrum of single- and multiexon NF1 copy number changes in a cohort of 1100 unselected NF1 patients. Genes Chromosomes Cancer 2006; 45: 265-276.

13 Ferec C, Casals T, Chuzhanova $\mathrm{N}$ et al: Gross genomic rearrangements involving deletions in the CFTR gene: characterization of six new events from a large cohort of hitherto unidentified cystic fibrosis chromosomes and meta-analysis of the underlying mechanisms. Eur J Hum Genet 2006; 14: 567-576.

14 Van Esch H, Bauters M, Ignatius J et al: Duplication of the MECP2 region is a frequent cause of severe mental retardation and progressive neurological symptoms in males. Am J Hum Genet 2005; 77: 442-453.

15 Thienpont B, Gewillig M, Fryns JP, Devriendt K, Vermeesch JR: Molecular cytogenetic characterization of a constitutional complex intrachromosomal 4q rearrangement in a patient with multiple congenital anomalies. Cytogenet Genome Res 2006; 114: 338-341. 